\title{
AN EVALUATION OF THE EPIDEMIOLOGY OF MEDICATION DISCREPANCIES AND CLINICAL SIGNIFICANCE OF MEDICINES RECONCILIATION IN CHILDREN ADMITTED TO HOSPITAL
}

Chi Huynh ${ }^{1,2}$, Stephen Tomlin ${ }^{3}$, Yogini Jani ${ }^{1,4}$, Guirish A. Solanki ${ }^{5}$, Helen Haley ${ }^{6}$, Rachel E. Smith ${ }^{7}$, Andrew Lowey ${ }^{7}$, Anthony Sinclair ${ }^{2}$, Keith A. Wilson ${ }^{8}$, Ian Chi Kei Wong ${ }^{1,9}$ David Terry ${ }^{2,8}$

${ }^{1}$ Centre for Paediatric Pharmacy Research, UCL School of Pharmacy, London UK;

${ }^{2}$ Academic Practice Unit, Pharmacy, Birmingham Children's Hospital, Birmingham UK;

${ }^{3}$ Evelina Children's Hospital, King's Health Partners, London, UK;

${ }^{4}$ Pharmacy Department, University College London Hospitals NHS Foundation Trust, London, UK;

${ }^{5}$ Neurosurgery Department, Birmingham Children's Hospital, Birmingham UK

'University Hospitals of North Midlands NHS Trust, UK;

${ }^{7}$ Leeds Children's Hospital, Leeds Teaching Hospital NHS Trust, Leeds UK;

${ }^{8}$ School of Pharmacy, Aston University, Aston Triangle, Birmingham UK;

${ }^{9}$ Department of Pharmacology and Pharmacy, Li Ka Shing Faculty of Medicine, University of Hong Kong. China.

Corresponding author: Dr David Terry

Address: - School of Pharmacy, Aston University, Aston Triangle, Birmingham UK

Email address: - d.terry@aston.ac.uk

\section{ABSTRACT}

\section{Aims}

To determine the incidence of unintended medication discrepancies in paediatric patients at the time of hospital admission; evaluate the process of medicines reconciliation; assess the benefit of medicines reconciliation in preventing clinical harm.

\section{Method:}

A five month prospective multisite study. Pharmacists at four English hospitals conducted admission medicines reconciliation in children using a standardised data collection form.

A discrepancy was defined as a difference between the patient's Pre-Admission Medication (PAM), compared with the initial Admission Medication Orders written by the hospital doctor. The discrepancies were classified into intentional and unintentional 
discrepancies. The unintentional discrepancies were assessed for potential clinical harm by a team of healthcare professionals, which included doctors, pharmacists and nurses.

\section{Results:}

Medicines reconciliation was conducted in 244 children admitted to hospital. $45 \%$ (109/244) of the children had at least one unintentional medication discrepancy between the Pre-Admission Medication and Admission Medication Order. The overall results indicated that $32 \%(78 / 244)$ of patients had at least one clinically significant unintentional medication discrepancy with potential to cause moderate $20 \%(50 / 244)$ or severe $11 \%$ (28/244) harm. No single source of information provided all the relevant details of a patient's medication history. Parents/carers provided the most accurate details of a patient's medication history in $81 \%$ of cases.

\section{Conclusion:}

This study demonstrates that in the absence of medicines reconciliation, children admitted to hospitals across England are at risk of harm from unintended medication discrepancies at the transition of care from the community to hospital. No single source of information provided a reliable medication history.

\section{Main manuscript \\ INTRODUCTION}

Medicines reconciliation is an intervention that reduces medication errors that occur at transitions in care for hospitalised patients.[1] Adult studies have shown that $60 \%$ of patients experience at least one unintended medication discrepancy upon admission or discharge, of which $18 \%$ have at least one clinically important discrepancy.[2] 
The UK has incorporated medicines reconciliation as a priority area for national patient safety, with national guidance in place for conducting medicines reconciliation in adults upon admission to hospital.[3] The guidance excluded children under the age of 16 years.[3]

Based on findings from a recent review of the literature regarding medication discrepancies at transitions in hospital care for children, there is limited evidence concerning the epidemiology of medication discrepancies across the interface of health care settings.[4] The review revealed only one UK study that evaluated the epidemiology of medication discrepancies at hospital admission and was limited to one neurosurgical ward at a children's hospital.[5] This study revealed that for 100 admissions, 39\% of medications written at hospital admission had a discrepancy when compared with the patient's pre-admission medication list, of which half had the potential to cause moderate or severe harm to the patient if left unresolved.[5] The review found that there were studies of medication discrepancies in North America which focused on medically complex children (USA) or was undertaken at a single site general medicine setting (Canada).[6,7] One particular study observed the accuracy of various sources of information to obtain an accurate medication history as an outcome measure.[8] Since the review was published, there has been one further published abstract observing the medication discrepancies of admission medications in Ireland. However this was also single site and had a small sample size. [9]

Based on the current limited evidence, this present study was conducted to determine if paediatric patients across England were at risk of clinically important discrepancies at hospital admission.[5] The primary objective was to prospectively identify the incidence of medication discrepancies that occurred at the time of hospital admission, prior to 
medicines reconciliation. The secondary objectives were to: evaluate the process of obtaining the medication history in terms of the time required for the process and the source and quality of information; and to assess the potential adverse clinical impact that unintended discrepancies identified may have, in the absence of medicines reconciliation.

\section{METHOD}

The study was conducted at paediatric hospital wards across all available specialities of four hospital sites providing secondary and tertiary care in Birmingham, Leeds, London and North Staffordshire in England.

The study cohort included children that were admitted over a 5 month study period, and prescribed at least one long-term medication. Long-term medication was defined as a medication that was prescribed for the child and expected to be taken on a repeat basis for three months or longer. Children were excluded from the study if: they were aged 19 years or older; the parent or carer was not available for interview; the drug chart was not accessible at the time of the data collection; or if the data collection could not be completed for practical reasons such as admission taking place out of hours in the evenings or during weekends (they would still receive routine care).

Medicines reconciliation was conducted by a clinical pharmacist who received training on using a pre-defined data collection form. Information concerning the child's diagnosis, age and weight, and speciality of the ward admitting the child were recorded. The following information retrieval processes were undertaken to support the medicines reconciliation:

- A semi-structured interview of the child's parent or carer to obtain a medication history and subsequently securing their permission to contact their community doctor, referred to as their General Practitioner (GP) in England 
- The determination of the child's medication history record, obtained by telephoning the child's GP practice

- Recording details of the Patient's Own Drugs (PODs) that were brought into hospital on admission

- Recording the initial Admission Medication Orders (AMOs) from the hospital drug chart prior to clinical pharmacist input

- A pharmacist determined regimen(s) was established based on the information gathered, and their clinical judgement as to what the patient should be prescribed in terms of long-term medications at the time of admission

The length of time it took to obtain the required information was also recorded.

Prior to the data collection, there was no specified procedure for medicines reconciliation in children. The approach to reconciliation in children, and sources used to verify the medication history was under the discretion and clinical decision of each clinical pharmacist.

For the purposes of identifying any discrepancies in the data collected, the patient's medication record from the GP was defined as the patient's Pre-Admission Medication (PAM) list, and this was compared against the initial Admission Medication Orders (AMOs) written by the hospital doctor prior to pharmacist input. When discrepancies were identified, the data collection pharmacist discussed each discrepancy with the prescriber. The discrepancies were further classified into intentional or unintentional discrepancies, as some of the charted medicines may have been intended changes according to clinical need after clarifying with the doctor. The classification of intentional and unintentional discrepancies was initially determined by the data collection pharmacist, usually in 
consultation with the prescriber, and later validated by a panel of clinical paediatric pharmacists from the four sites.

The clinical significance of the unintentional discrepancies identified was assessed using the methodology used by Terry et al adapted from Cornish et al.[5,10]

A panel of experts consisting of two paediatric clinical pharmacists, two hospital doctors and a medicines management nurse (the "Clinical Assessment Panel") met and categorised the discrepancies. Each discrepancy was considered on a case by case basis. The patient's age and diagnosis were provided to the panel.

The Clinical Assessment Panel was asked to rank each discrepancy according to potential harm using three ordinal classifications based on a theoretical scenario where the discrepancies were left unchanged over a period of 7 days: Class 1: - Unlikely to cause patient discomfort or clinical deterioration; Class 2: - Potential to cause moderate discomfort or clinical deterioration; Class 3: - Potential to result in severe discomfort or clinical deterioration.[10]

The incidence of the unintentional discrepancies was calculated as: -

$$
\frac{\text { number of patients with at least one unintentional discrepancy }}{\text { total number of patients }}
$$

The incidences of unintentional discrepancies per patient within each of the three classifications 1, 2 or 3 were calculated as: -

$$
\frac{\text { number of patients with class } 1 \text { ( } 2 \text { or } 3 \text { ) discrepancies }}{\text { total number of patients }}
$$

Any individual patient could have more than one classification of discrepancy. Therefore a further incidence value was calculated based on the number of patients who had class 1,2 or 3 expressed as their most serious discrepancy. This was calculated as: - 
number of patients with a class 1 (2 or 3 ) discrepancy as the most serious discrepancy total number of patients

In addition to collecting information relevant to the medication history, the time to complete each process was recorded to determine the time resource required to conduct medicines reconciliation. Once information was collected from all the sources and the pharmacist determined regimen was established, the sensitivity of each source of information against the pharmacist determined regimen was calculated.

\section{Ethical consideration}

This study was deemed a service evaluation project by the Research \& Development Department of all sites involved and therefore NHS ethics committee approval was not required. Although NHS ethics was not required, this project was approved by the School of Pharmacy, University of London Ethics Committee in 2011. All data collected were anonymised and stored appropriately according to NHS information governance procedures.

\section{RESULTS}

244 patients were admitted to the study and 1004 individual drug prescriptions were recorded. The age of the patients ranged from 1 month to 16 years of age (median age of 5 years, Inter-Quartile Range IQR 1.5 years -11.25 years). The most common speciality associated with the admissions were, general paediatric medicine $(n=80)$, respiratory $(n=$ 35 ) and surgery $(n=31)$. See Table 1 for full details of the number of admissions per speciality.

The data collection pharmacists identified 582 medication discrepancies from the 1004 drug prescriptions (58\%) affecting 203 patients (83\%). Of the 582 discrepancies, 209 
were classified as unintentional, 277 were intentional and 96 were reclassified as either trivial, relating to nutritional feeds rather than medicines or other (other being discrepancies that the team were unable to classify because the drug regimen was not on the GP record, nor written up on the hospital drug chart but identified by the pharmacist or patient).

The 209 unintentional discrepancies, affecting 109/244 (45\%) patients, were classified using the method by Terry and colleagues. It was found that 189 drug discrepancies affecting 100 patients were unintentional discrepancies and were classifiable into the "harm" classifications. In the view of the 'Clinical Assessment Team' the remaining 20 unintentional discrepancies were likely to be clinically beneficial to the patient and therefore were excluded from classification of harm. Nine patients were identified who only had potentially beneficial discrepancies were excluded in the clinical assessment for harm. The incidence of each class of discrepancy was: $22 \%$ (22/100) patients with Class 1 (Unlikely to cause patient discomfort or clinical deterioration); 50\% (50/100) patients with Class 2 (Potential to cause moderate discomfort or clinical deterioration) and 28\% (28/100) patients with Class 3 (Potential to result in severe discomfort or clinical deterioration).

The overall results indicated that $32 \%(78 / 244)$ of patients in the study had at least one clinically significant unintentional discrepancy, rated as moderate Class $2(50 / 244)$ or severe Class $3(28 / 244)$ potential harm. Please see Table 2 for selected examples highlighting Class 1, Class 2 and Class 3 discrepancies.

The total time required for completing medicines reconciliation for each of the 244 study patients ranged from 6 to 144 minutes (Median 24, Inter-quartile range $17-40$ ). 
A comparison of the pharmacist determined regimen (a decision the pharmacist made at the conclusion of the medicines reconciliation process) against the sources of information used showed that the parent-carer provided the most accurate information by matching $81 \%$ of the pharmacist determined regimens. Parents were able to identify all their child's medication in $71 \%$ of admissions, with $15 \%$ being able to identify some but not all of their child's medication regimens based on a comparison against the pharmacist determined regimen. The pre-admission medication list from the GP matched the pharmacist determined regimen in $70 \%$ of cases. Patient's own drugs (PODs) were only present in 94 admissions and only $56 \%$ (53/94) of PODs that were available at admission were of use and matched the pharmacist determined regimen. In $7.4 \%$ of cases, there were conflicting information between all the sources of information gathered and the pharmacist made the recommendation based on clinical judgement and a review of the patient notes.

\section{DISCUSSION}

This study found that medication discrepancies occurred at hospital admission when admission medication orders were written by prescribers in the absence of medicines reconciliation. Almost half of patients who were admitted to hospital on at least one long term medication experienced one or more unintended medication discrepancies. Of this cohort one in five patients were at risk of moderately (class 2 ) clinical harm and one in ten were at risk of serious clinical harm (class 3 ). The findings of this present study add to current evidence that in the absence of medicines reconciliation, medication discrepancies occur across the paediatric population in England. As this study was carried out across four geographically different hospital settings with variations in health service delivery, the findings may be generalizable to the paediatric population nationally and internationally, 
but further investigations are required to confirm this. A later study that was conducted in a paediatric hospital setting in Ireland found that $37.5 \%$ of patients (15 out of 40 patients) from a single hospital site in Ireland had at least one undocumented discrepancy on their drug Kardex. Further details of whether or not these undocumented discrepancies were intended or unintended were not provided in the abstract report.[9] Although the sample size for the study in Ireland was small, the comparable rates suggest that the results of our study may be generalizable.

Two additional outcome measures were evaluated during the course of our study. The first was the sensitivity of each source of information and it was found that the parent/carer was the most sensitive (accurate) source of information, followed by the GP and the patient own drugs (PODs). PODs were seen as a less accurate source since they are infrequently brought into hospital and thus by default will not give all the data required. The finding that the parents were the most reliable source of information supports previous studies observing the information sources that made up a medication history in a child [8]. However a study sited in USA observed that approximately half of parents were not available within the first 18 hours of admission in medically complex children [6], although this was not apparent in our study. Many parents expressed the dose of liquid medicines in terms of volume alone (e.g. millilitres). This highlights the importance of a second source of information, either the GP of POD, to confirm the actual dose required (e.g. milligrams). This is particularly important when prescribing for children due to the variety of unlicensed preparations available. An important finding is that the GP who is responsible for the care of the patient outside hospital did not hold all the information regarding the patient's current medication. These findings demonstrate the complexity of correctly identifying medicines for children; who are often cared for via several health care providers and may have several carers and care settings. 
The second outcome measured was the time taken to complete medicines reconciliation using the data collection forms. It was found that the median time taken to conduct medicines reconciliation was twenty-four minutes per patient and the longest time was two hours and twenty-four minutes. This average value was lower than the estimated time reported by a study of medicines reconciliation carried out in medically complex children in the USA which was 90 minutes.[6] The shorter median time may be as the present study included all patients who were on at least one long-term medication prior to hospital admission and did not restrict or impose criteria to prioritise children with complex medication regimens.

As with all studies that have observed medication 'discrepancies' at hospital admission, there was no single exact or perfect list of medications that a patient was taking prior to admission (sometimes referred to as a 'gold standard'). Unlike other observational studies rather than using a "best possible medication history" list [7] or 'verified outpatient list' [6], this study utilised the GP as the main reference point to identify pre-admission medication. This decision was made during methodology development as the GP was expected to be the main gatekeeper of information and hold information on all medications the patient was prescribed. However, this study demonstrates the limitation of GP held information.

This study confirmed that clinically significant medication discrepancies may occur in hospitalised children. This is not isolated to one particular speciality or hospital. Medicines reconciliation is important in detecting and rectifying these discrepancies. Existing studies have explored the completeness of sources of information to obtain medication histories [8], or monitored the uptake of medicines reconciliation. [11] However, to the best of our knowledge, no studies have been published concerning the design of evidence based and user evaluated medicines reconciliation interventions for children. 


\section{CONCLUSION}

This study has demonstrated that children who are admitted to hospitals across England are at risk of harm from unintended medication discrepancies in the absence of medicines reconciliation. Of the four sources of information adopted by this study, not one source of information could provide a complete medication list, which is similar to studies in adult patients. This finding and the fact that the method used in the study was not common practice at the study sites highlights that a more thorough approach of conducting medicines reconciliation in the paediatric population is required. Further work is required to design and implement a practical and efficient intervention to reduce harm and standardise medicines reconciliation.

\begin{tabular}{|l|}
\hline What is already known on this topic \\
pedication discrepancies have been shown to occur at hospital admission for \\
Current studies are limited to individual specialties, single hospital sites, and small \\
patient samples. \\
\hline What this study adds \\
Medication discrepancies can occur in paediatric patients who are on long term \\
medication, and may cause potentially harmful consequences. \\
Medicines reconciliation can resolve potentially harmful unintended medication \\
discrepancies in children.
\end{tabular}

\section{REFERENCES}

[1] WHO Collaborating Centre for Patient Safety Solutions. Assuring Medication Accuracy at Transitions in Care. Geneva: WHO, 2007. 
[2] Vira T, Colquhoun M, Etchells E. Reconciliable differences: correcting medication errors at hospital admission and discharge. Quality and Safety in Health Care 2006;15:122-126.

[3] National Institute for Health and Clinical Excellence. National Patient Safety Agency. PSG001. Technical patient safety solutions for medicines reconciliation on admission of adults to hospital. London: NICE; 2007

[4] Huynh C, Wong ICK, Tomlin S, et al. Medication discrepancies at transitions in pediatrics: a review of the literature. Pediatric Drugs 2013;15(3):203-215.

[5] Terry D, Solanki G, Sinclair A, et al. Clinical significance of medication reconciliation in Children admitted to a UK paediatric hospital. Pediatric Drugs 2010;12(5):331-337.

[6] Stone BL, Boehme S, Mundorff MB, et al. Hospital admission medication reconciliation in medically complex children: an observational study. Archives of Diseases in Childhood 2010;95:250-56.

[7] Coffey M, Mack L, Streitenberger K, et al. Prevalence and clinical significance of medication discrepancies at pediatric hospital admission. Academic Pediatrics 2009;9: 360-366.

[8] Dersch-Mills D, Hugel K, Nystrom M. Completeness of information sources used to prepare best possible medication histories for pediatric patients. Canadian Journal of Hospital Pharmacy 2011;64(1):10-15.

[9] Butler E, Bourke C. Medicine reconciliation on admission in a paediatric hospital setting. Archives of Diseases in Childhood 2013;98:e1

[10] Cornish PL, Knowles SR, Marchesano R, et al. Unintended medication discrepancies at the time of hospital admission. Archives in Internal Medicine 2005;165:424-423

[11] Coffey M, Cornish P, Koonthanam T, et al. Implementation of admission medication reconciliation at two academic health sciences centres: Challenges and success factors. Healthcare Quarterly 2009;12:102-109.

\section{LICENCE STATEMENT}

The Corresponding Author has the right to grant on behalf of all authors and does grant on behalf of all authors, an exclusive licence (or non-exclusive for government employees) on a worldwide basis to the BMJ and co-owners or contracting owning societies (where published by the BMJ on their behalf), and its Licensees to permit this article (if accepted) 
to be published in Archives of Disease in Childhood and any other BMJ products and to exploit all subsidiary rights, as set out in our licence.

\section{CONTRIBUTORSHIP STATEMENT}

Chi Huynh was responsible for the acquisition of data across one site, analysis and interpretation of the complete dataset across the four sites, prepared the manuscript of the study

Stephen Tomlin contributed towards the conception of the study design, reviewing the interpretation of data and was involved in approving the final version of the manuscript published.

Yogini Jani contributed towards reviewing the interpretation of data and was involved in approving the final version of the manuscript published.

Guirish A. Solanki contributed towards reviewing the interpretation of data and was involved in approving the final version of the manuscript published.

Helen Haley contributed towards the conception of the study design, contributed towards the acquisition of data across one of the four sites, and was involved in approving the final version of the manuscript published.

Rachel E. Smith contributed towards the acquisition of data across one of the four sites and was involved in approving the final version of the manuscript published.

Andrew Lowey contributed towards the conception of the study design, and was involved in approving the final version of the manuscript published. 
Anthony Sinclair contributed towards the conception of the study design, and was involved in approving the final version of the manuscript published.

Keith Wilson contributed towards the conception of the study design, and was involved in approving the final version of the manuscript published.

Ian Chi Kei Wong contributed towards the conception of the study design, and was involved in approving the final version of the manuscript published.

David Terry contributed towards the conception of the study design, the acquisition of data across one of the four sites and was involved in approving the final version of the manuscript published.

\section{COMPETING INTERESTS}

None declared

CONFLICT OF INTEREST

None declared

\section{FUNDING}

This work was supported by a grant from the Neonatal and Paediatric Pharmacists Group (No grant number issued). 